\title{
Estrogen restricts the apoptosis of endometrial stromal cells by promoting TSLP secretion
}

\author{
HUI-LI YANG $^{1 *}$, KAI-KAI CHANG ${ }^{2 *}$, JIE MEI $^{3}$, WEN-JIE ZHOU ${ }^{1,4}$, LI-BING LIU ${ }^{5}$, LI YAO ${ }^{1}$, YI MENG ${ }^{6}$, \\ MING-YAN WANG ${ }^{6}$, SI-YAO HA ${ }^{1}$, ZHEN-ZHEN LAI ${ }^{1}$, JIANG-FENG YE ${ }^{7}$, DA-JIN LI ${ }^{1}$ and MING-QING LI ${ }^{1,4}$ \\ ${ }^{1}$ Laboratory for Reproductive Immunology, Key Laboratory of Reproduction Regulation of NPFPC, SIPPR, IRD, \\ Hospital of Obstetrics and Gynecology, Fudan University Shanghai Medical College, Shanghai 200080; \\ ${ }^{2}$ Department of Gynecology, Hospital of Obstetrics and Gynecology, Fudan University, Shanghai 200011; \\ ${ }^{3}$ Department of Obstetrics and Gynecology, Reproductive Medicine Center, Nanjing Drum Tower Hospital, \\ The Affiliated Hospital of Nanjing University Medicine School, Nanjing, Jiangsu 210000; \\ ${ }^{4}$ Shanghai Key Laboratory of Female Reproductive Endocrine Related Diseases, \\ Hospital of Obstetrics and Gynecology, Fudan University Shanghai Medical College, Shanghai 200011; \\ ${ }^{5}$ Department of Gynecology, Changzhou No. 2 People's Hospital, Affiliated with Nanjing Medical University, \\ Changzhou, Jiangsu 213003; Departments of ${ }^{6}$ Clinical Laboratory and ${ }^{7}$ Clinical Epidemiology, \\ Hospital of Obstetrics and Gynecology, Fudan University, Shanghai 200011, P.R. China
}

Received March 19, 2018; Accepted July 27, 2018

DOI: $10.3892 / \mathrm{mmr} .2018 .9428$

\begin{abstract}
Endometriosis (EMS) is a female hormonedependent disease with controversial reports of its etiology and pathogenesis. Apoptosis is particularly important in the human endometrium due to the dynamic cycles of proliferation and shedding. Estrogen possessed antiapoptotic effects on endometrial stromal cells (ESCs), which appears to be exacerbated in women with EMS; however, the underlying mechanism of the antiapoptotic effects of estrogen on ESC remains unknown. The present study aimed to determine whether estrogen regulates the apoptosis of ESCs via thymic
\end{abstract}

Correspondence to: Professor Ming-Qing Li, Laboratory for Reproductive Immunology, Key Laboratory of Reproduction Regulation of NPFPC, SIPPR, IRD, Hospital of Obstetrics and Gynecology, Fudan University Shanghai Medical College, 1326 Pingliang Road, Shanghai 200080, P.R. China

E-mail:mqli@fudan.edu.cn

*Contributed equally

Abbreviations: EMS, endometriosis; ESCs, endometrial stromal cells; TSLP, thymic stromal lymphopoietin; Bcl-2, B-cell lymphoma; rhTSLP, recombinant human TSLP; $\alpha$ TSLP, anti-human TSLP neutralizing antibody; TSLPR, TSLP receptor; $\mathrm{E}_{2}, 17-\beta$ estradiol; ER, estrogen receptor; IL, interleukin; ELISA, enzyme linked immunosorbent assay; FCM, flow cytometry; DMEM/F12, Dulbecco's modified Eagle's medium/F12; FBS, fetal bovine serum; PI, propidium iodide

Key words: estrogen, apoptosis, thymic stromal lymphopoietin, B cell lymphoma 2, endometrial stromal cells, endometriosis stromal lymphopoietin (TSLP) and the associated mechanism. An ELISA was conducted to detect TSLP content in the ESC culture medium treated with estrogen. Subsequently, the early apoptotic rate and expression of B-cell lymphoma (Bcl-2) of ESCs were analyzed by flow cytometry in the presence of recombinant human TSLP, anti-human TSLP neutralizing antibody or estrogen. In the present study, it was reported that ESCs exhibited basal TSLP secretion in the absence of estrogen as reported in previous studies, and that estrogen promoted TSLP secretion of ESCs in a dose-dependent manner. The results demonstrated that estrogen suppressed the apoptosis of ESCs associated with the promotion of Bcl-2 expression, which may be partly reversed by inhibiting TSLP. Therefore, the findings of the present study revealed a novel mechanism of estrogen-dependent apoptotic suppression of ESCs associated with TSLP secretion and Bcl-2 regulation. Endogenous and estrogen-induced endometrial TSLP may promote the initiation and development of EMS via the inhibition of apoptosis.

\section{Introduction}

Endometriosis (EMS), a chronic inflammatory disease among women of reproductive age (age, 15-49 years old), is defined as the presence of endometrial-like tissues outside the uterus (1). The theory of retrograde menstruation suggests that shed endometrial fragments may pass back into the peritoneal cavity via the fallopian tubes, initiating the formation of endometriotic lesions (2).

In the human endometrium, apoptosis is particularly important due to the dynamic cycles of shedding and proliferation (3); ectopic endometrial cells have been reported to exhibit abnormal proliferative and apoptotic regulation in response to appropriate stimuli (4). At present, the reduced susceptibility of endometriotic epithelial and stromal cells to apoptosis has 
been considered to contribute to the pathogenesis of EMS (5). Apoptosis in the eutopic endometrium of women with EMS and endometriotic lesions was observed to be lower than that of the the normal endometrium of women without EMS (5).

It has been well reported that EMS is a hormone-responsive disease associated with increased estrogen production and resistance against the progesterone-based response $(6,7)$. Increased expression levels of estrogen receptors (ER) $\alpha$ and $\beta$ have been observed in ectopic tissue compared with in normal and eutopic endometrium (8). In addition, increased estrogen availability in EMS was reported to lead to notable protein kinase activation and apoptosis inhibition (9).

Thymic stromal lymphopoietin (TSLP) is produced by stromal cells, epithelial cells, fibroblasts, basophils, keratinocytes and other cells types $(10,11)$. TSLP was reported to stimulate the growth and activation of B cells; however, analysis has demonstrated that TSLP affects the hematopoietic and non-hematopoietic cell lineages (12); endometrial stromal cells (ESCs) can synthesize and secrete TSLP (13). Additionally, interleukin (IL)-1 $\beta$ was reported to increase the expression of TSLP mRNA and secretion within ESCs, which can be promoted by IL-4 (13). Our previous study revealed that estrogen can increase the production of TSLP in ESCs in a dose-dependent manner, and that ESC-derived and exogenous TSLP can activate the secretion of ESC-associated growth-promoting cytokines (monocyte chemotactic protein-1 and IL-8) and promote the viability and proliferation of ESCs (14).

The mechanism underlying the inhibition of apoptosis mediated by estrogen in EMS is not completely clear; whether estrogen regulates the apoptosis of ESCs by modulating the expression of TSLP requires further investigation. Therefore, the present study aimed to investigate the effects of the estrogen-TSLP axis on the inhibition of EMS-associated apoptosis.

\section{Materials and methods}

Tissues. To study how estrogen regulates the apoptosis of ESCs in EMS, we collected ectopic endometrium from subjects with EMS and obtained ectopic ESCs from hyperoestrogenic environment rather than normal ESCs from women without EMS. They may have distinctive characteristics. Participants were women attending the Obstetrics and Gynecology Hospital of Fudan University between May and December 2017. Ectopic endometrial tissues were obtained from fertile women (age, 21-46 years old) with ovarian $(n=25)$ and pelvic $(n=5)$ EMS. Clinical suspicion of endometriosis was based upon patient symptoms including dysmenorrhea, deep dyspareunia, chronic pelvic pain, infertility and cyclical alterations in bowel and urinary habits occurring only during menstruation. After physical examination, patients were subjected to transvaginal ultrasound or MRI. According to the suspicion of the presence of deep infiltrating lesions or if the patient had persistent pain or infertility, a surgical laparoscopic procedure was indicated. During laparoscopy, biopsies from ectopic lesions were obtained from each patient. Based on histopathology and medical records, patients with endometrioma (OMA), superficial peritoneal endometriosis (SPE), and deep infiltrating endometriosis (DIE) were included, however, adenomyosis or pelvic inflammatory disease (PID) related infertility were excluded. All samples were obtained in the proliferative phase of the endometrial cycle, which were confirmed histologically according to established criteria $(15,16)$. None of the women had received hormonal medication in the 3 months prior to surgery. The collection of tissue samples was conducted following the obtainment of informed consent from patients and was in accordance with the requirements of the Research Ethics Committee of the Hospital of Obstetrics and Gynecology, Fudan University (Shanghai, China).

Culture of ESCs. The ectopic lesion tissues were collected under sterile conditions and were transported to our laboratory (Laboratory for Reproductive Immunology, Hospital of Obstetrics and Gynecology, Fudan University Shanghai Medical College) on ice in Dulbecco's modified Eagle's medium (DMEM)/F-12 (HyClone; GE Healthcare Life Sciences, Logan, UT, USA). All tissues were minced into $2 \mathrm{~mm}$ pieces and then digested with $20 \%$ collagenase type IV (0.1\%; Sigma-Aldrich; Merck KGaA, Darmstadt, Germany) for $40 \mathrm{~min}$ at $37^{\circ} \mathrm{C}$ with constant agitation. The tissue pieces were filtered via sterile gauze pads (pore diameter sizes: 100, 200 and 400 mesh) to remove cellular debris and separate the ESCs from epithelial cells. The filtrate was then centrifuged at $150 \mathrm{x} \mathrm{g}$ at $4^{\circ} \mathrm{C}$ for $8 \mathrm{~min}$ and washed with PBS. Following removal of the supernatant, the cells were resuspended in DMEM/F-12 containing 10\% fetal bovine serum (FBS; Sigma-Aldrich; Merck KGaA), plated in culture flasks and incubated in a humidified incubator with $5 \% \mathrm{CO}_{2}$ at $37^{\circ} \mathrm{C}$. The culture medium was replaced every 2-3 days. These methods supplied $>95 \%$ Vimentin-positive $\left(\right.$ Vimentin $\left.^{+}\right)$cytokeratinnegative (cytokeratin') ESCs as demonstrated previously (17).

ELISA. ESCs were seeded at $1 \times 10^{5}$ cells/well in 24-well plates and treated with various concentrations of $17-\beta$ estradiol $\left(\mathrm{E}_{2} ; 10^{-9} \mathrm{M}, 10^{-8} \mathrm{M}\right.$ or $10^{-7} \mathrm{M}$; Sigma-Aldrich; Merck KGaA) in phenol red-free DMEM (HyClone; GE Healthcare Life Sciences) containing 10\% dextran-coated charcoal-treated FBS (Sigma-Aldrich; Merck KGaA), and incubated for $48 \mathrm{~h}$ with $5 \% \mathrm{CO}_{2}$ at $37^{\circ} \mathrm{C}$. The controls were treated with $0.1 \%$ dimethyl sulfoxide (DMSO; Sigma-Aldrich; Merck KGaA). After 48 h of culture, the culture supernatant was harvested via centrifugation at $150 \mathrm{x} \mathrm{g}$ at $4^{\circ} \mathrm{C}$ for $8 \mathrm{~min}$ to remove cellular debris, and stored at $-80^{\circ} \mathrm{C}$. Then an ELISA (cat. no. DTSLP0; R\&D Systems Europe, Ltd., Abingdon, UK) was conducted according to the manufacturer's protocol to determine the expression of TSLP in the supernatant of the samples.

Treatment of ESCs. ESCs were cultured in 24-well plates at $1 \times 10^{5}$ cells/well and treated with various concentrations of recombinant human TSLP (rhTSLP; 1, 10 or $100 \mathrm{ng} / \mathrm{ml}$; cat. no. 1938-TS, R\&D Systems Europe, Ltd.) for $48 \mathrm{~h}$; the control group was treated with $0.1 \%$ PBS. ESCs in TSLP-treated group or control group were cultured in DMEM/F-12 containing $10 \%$ fetal bovine serum (FBS; Sigma-Aldrich; Merck $\mathrm{KGaA}$ ) and incubated in a humidified incubator with $5 \% \mathrm{CO}_{2}$ at $37^{\circ} \mathrm{C}$ for $48 \mathrm{~h}$.

In addition, ESCs under the same culture conditions were treated with $\mathrm{E}_{2}\left(10^{-7} \mathrm{M}\right)$ alone, anti-human TSLP neutralizing antibody ( $\alpha$ TSLP; $0.25 \mu \mathrm{g} / \mathrm{ml}$; cat. no. AF1398, R\&D Systems Europe, Ltd.) alone or $\mathrm{E}_{2}$ and $\alpha \mathrm{TSLP}$ for $48 \mathrm{~h}$ at $37^{\circ} \mathrm{C}$ 


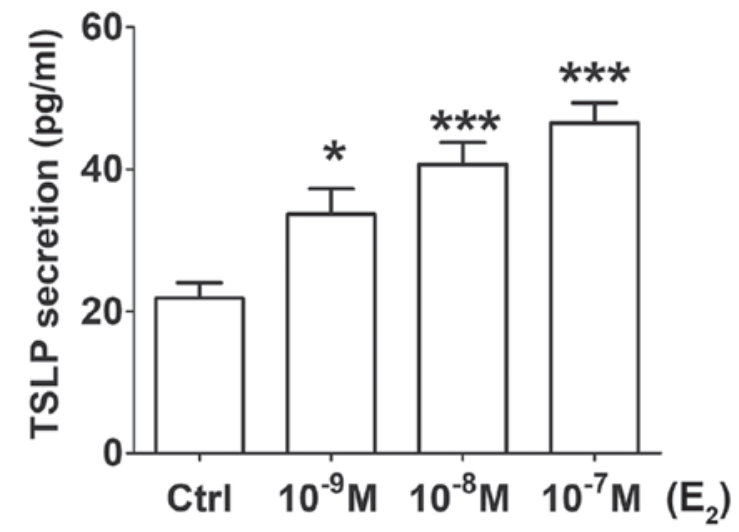

Figure 1. Estrogen promotes TSLP secretion of ESCs in a dose-dependent manner. Following treatment with or without various concentration of $E_{2}$ $\left(10^{-9}, 10^{-8}\right.$ and $\left.10^{-7} \mathrm{M}\right)$ for $48 \mathrm{~h}$, the levels of TSLP secretion of ESCs were detected by ELISA. The data are expressed as the mean \pm standard error of the mean. ${ }^{*} \mathrm{P}<0.05$ and ${ }^{* * * *} \mathrm{P}<0.001$ vs. the $\mathrm{Ctrl}$ group. $\mathrm{Ctrl}$, control; $\mathrm{E}_{2}, 17-\beta$ estradiol; ESCs, endometrial stromal cells from patients with endometriosis; TSLP, thymic stromal lymphopoietin.

with $5 \% \mathrm{CO}_{2}$. The concentration of $\mathrm{E}_{2}$ was $10^{-7}$ for a better stimulation of TSLP secretion of ESCs (Fig. 1). The controls were treated with $0.1 \%$ DMSO for $48 \mathrm{~h}$ at $37^{\circ} \mathrm{C}$ with $5 \% \mathrm{CO}_{2}$. The ESC groups were employed for apoptosis analysis and flow cytometry (FCM) as described below.

Apoptosis analysis. Apoptotic cell death was detected via Annexin V-fluorescein isothiocyanate (FITC)/propidium iodide (PI) staining using the apoptosis kit (Annexin V FITC Apop Dtec kit, BD Biosciences, Franklin Lakes, NJ, USA). ESCs $\left(1 \times 10^{5}\right.$ cells) from the various cultures were trypsinized using $0.25 \%$ Trypsin (1x, Phenol Red; no EDTA; Gibco, Grand Island, New York, NY, USA) for $3 \mathrm{~min}$ at $37^{\circ} \mathrm{C}$ with $5 \% \mathrm{CO}_{2}$ and collected, washed and resuspended in $300 \mu$ l binding buffer included in the apoptosis kit, followed by incubation with $5 \mu \mathrm{l}$ Annexin V-FITC and $2.5 \mu \mathrm{l}$ PI at room temperature for $15 \mathrm{~min}$ in the dark. Then, $200 \mu \mathrm{l}$ binding buffer was added and the cell samples were analyzed with a Beckman CyAn flow cytometer (Beckman Coulter, Inc., Brea, CA, USA) using Becton Dickinson CellQuest software (version 7.1; Becton Dickinson). Annexin $\mathrm{V}^{+} \mathrm{PI}^{-}$cells were in the early stage of apoptosis and Annexin $\mathrm{V}^{+} \mathrm{PI}^{+}$cells were late apoptotic cells. The percentage of the early apoptotic cells was determined as the apoptosis rate. The experiments were performed in triplicate.

$F C M$. The ESCs $\left(1 \times 10^{5}\right.$ cells) in the various treatment groups were centrifuged immediately at $150 \mathrm{xg}$ at $4^{\circ} \mathrm{C}$ for $8 \mathrm{~min}$. The expression of B- cell lymphoma 2 (Bcl-2), Fas and Fas ligand (FasL) was analyzed by FCM. These ESCs were fixed use $0.5 \mathrm{ml}$ Fixation Buffer (Biolegend, Inc., San Diego, CA, USA) in the dark for $20 \mathrm{~min}$ at room temperature. For permeabilization, we resuspend fixed cells in diluted Intracellular Staining Permeabilization Wash Buffer (Biolegend, Inc., San Diego, CA, USA) and centrifuged at $350 \mathrm{x}$ g for 5-10 min, and repeated the process twice at room temperature. Staining was performed with Brilliant Violet ${ }^{\mathrm{TM}}$ 421-conjugated anti-human Bcl-2 (cat. no. 658709), allophycocyanin-conjugated anti-human Fas (cat. no. 305611) and phycoerythrin-conjugated anti-human
FasL (cat. no. 306406) antibodies (5 $\mu \mathrm{l}$ separately, all antibodies were obtained from Biolegend, Inc., San Diego, CA, USA) at room temperature for $30 \mathrm{~min}$ in the dark. In addition, isotypic IgG antibodies ( $5 \mu \mathrm{l}$ separately), including Brilliant Violet $^{\mathrm{TM}} 421$ anti-mouse IgG1 antibody (cat. no. 406615), APC anti-mouse IgG1 antibody (cat. no. 406609) and PE anti-mouse IgG1 antibody (cat. no. 406607) were used as controls. Subsequently, the cells were washed twice and resuspended in PBS for FCM analysis. Samples were analyzed with a Beckman Cyan flow cytometer (Beckman Coulter, Inc.) using CellQuest software (version 7.1; Beckman Coulter, Inc.). The experiments were performed in triplicate and repeated three times.

Statistical analysis. All data were analyzed via one-way analysis of variance, followed by a Tukey post-hoc test for the comparison of multiple groups using SPSS software, version 11.5 (SPSS, Inc., Chicago, IL, USA). Each experiment was conducted three times and results are presented as the mean \pm standard error. $\mathrm{P}<0.05$ was considered to indicate a statistically significant difference.

\section{Results}

Estrogen promotes TSLP secretion of ESCs. Following $48 \mathrm{~h}$ of incubation, the concentration of TSLP in the ESC culture supernatant was significantly higher with increased concentrations of $\mathrm{E}_{2}\left(10^{-9}, 10^{-8}\right.$ or $\left.10^{-7} \mathrm{M}\right)$ (Fig. 1) compared with in the control group. ESCs obtained from the ectopic lesions from patients with EMS exhibited basal secretion levels of TSLP as $>20 \mathrm{pg} / \mathrm{ml}$ TSLP was detected in the control group (treated with $0.1 \%$ DMSO), which was consistent with previous studies $(13,14)$. Treatment with $10^{-9} \mathrm{M} \mathrm{E}_{2}$ resulted in a significant increase of TSLP secretion $(\mathrm{P}<0.05)$; upon administration of $10^{-8}$ and $10^{-7} \mathrm{M} \mathrm{E}_{2}$, the concentration of TSLP in the supernatant was increased by $\sim 2$-fold compared with in the control $(\mathrm{P}<0.001)$ (Fig. 1). The results suggested that estrogen promoted TSLP secretion of ESCs in a dose-dependent manner.

TSLP inhibits the apoptosis of ESCs. In order to investigate the how TSLP affects the apoptosis of ESCs, increasing concentrations of rhTSLP was applied to ESCs in vitro obtained from the ectopic lesions of patients with EMS. After $48 \mathrm{~h}$ following treatment, ESCs were obtained for FCM analysis to detect the rate of apoptosis. The administration of rhTSLP resulted in decreases in the number of apoptotic ESCs (Fig. 2A). Analysis of the early apoptotic rate demonstrated that $1 \mathrm{ng} / \mathrm{ml}$ rhTSLP significantly reduced the early apoptotic rate $(\mathrm{P}<0.05)$; however, highly significant reductions in apoptosis were reported in response to 10 and $100 \mathrm{ng} / \mathrm{ml}(\operatorname{rhTSLP}(\mathrm{P}<0.01$; $\mathrm{P}<0.001$ ) (Fig. 2B). These results suggested that exogenous TSLP could inhibit the apoptosis of ESCs in vitro.

TSLP increases the number of $\mathrm{Bcl}-2^{+}$ESCs. Following the treatment ESCs with rhTSLP for $48 \mathrm{~h}$, cells expressing the antiapoptotic protein $\mathrm{Bcl}-2$, and two apoptosis-associated markers, Fas and FasL were detected by FCM. As the concentration of TSLP increased, the proportion of $\mathrm{Bcl}-2^{+}$ESCs also increased (Fig. 3A); a significant difference was observed in response to treatment of the highest concentration $(100 \mathrm{ng} / \mathrm{ml})$ of rhTSLP 
A rhTSLP

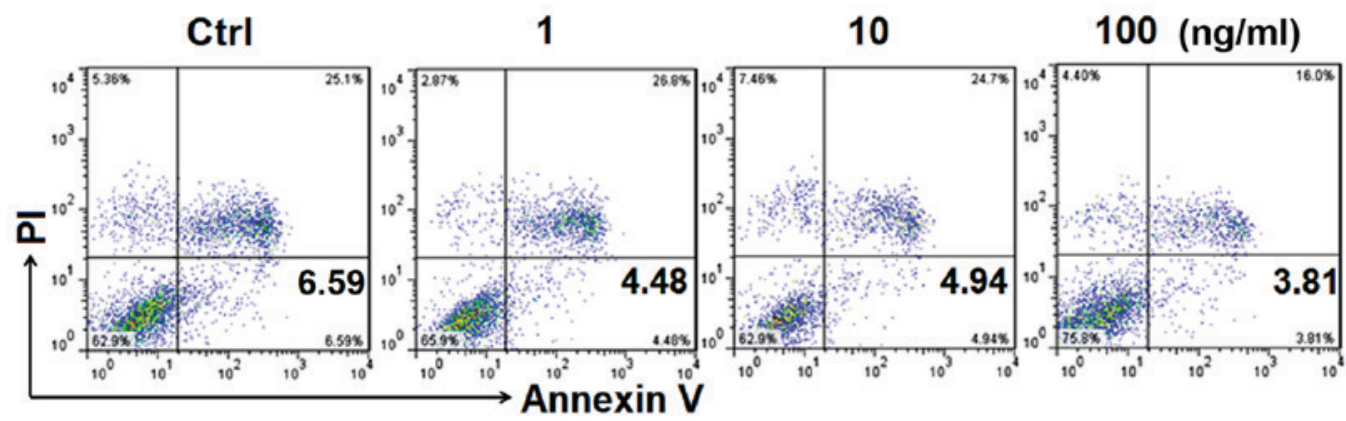

B

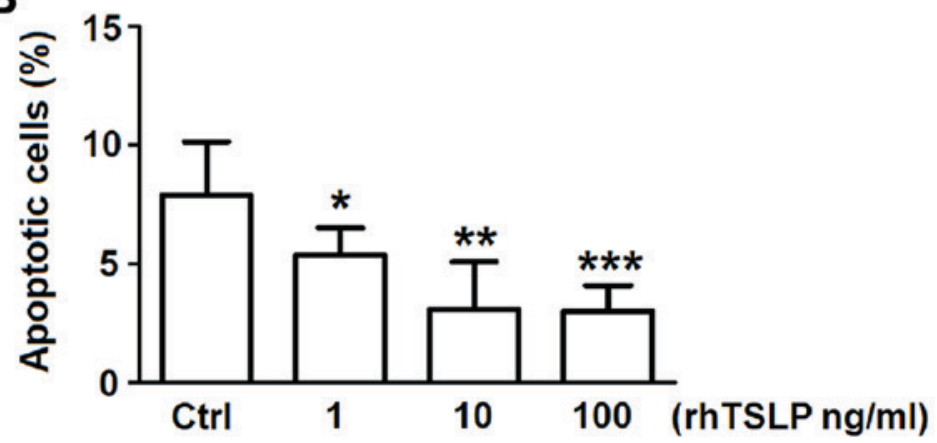

Figure 2. TSLP inhibits the apoptosis of ESCs. (A) Early apoptotic analysis (\% of Annexin $\mathrm{V}^{+} \mathrm{PI}$ ESCs) of ESCs treated with rhTSLP $(0,1,10 \mathrm{or} 100 \mathrm{ng} / \mathrm{ml})$ for $48 \mathrm{~h}$ was analyzed by flow cytometry. (B) Statistical analysis about the rate of early apoptosis of flow cytometry. The data are expressed as the mean \pm standard error of the mean. ${ }^{*} \mathrm{P}<0.05,{ }^{* *} \mathrm{P}<0.01$ and ${ }^{* * *} \mathrm{P}<0.001$ vs. the Ctrl group. Ctrl, control; ESCs, endometrial stromal cells from patients with endometriosis; PI, propidium iodide; rhTSLP, recombinant human thymic stromal lymphopoietin.

A

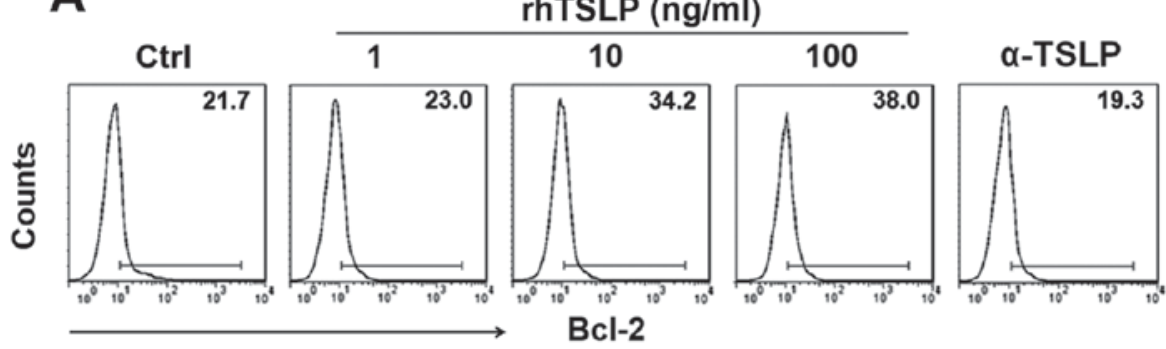

B

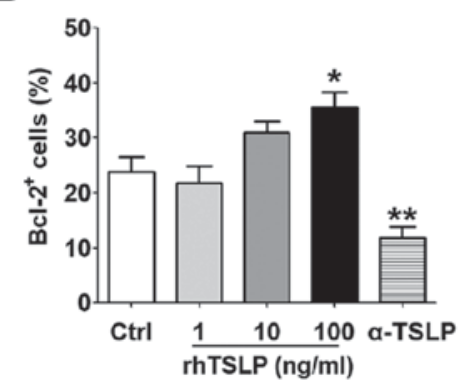

C

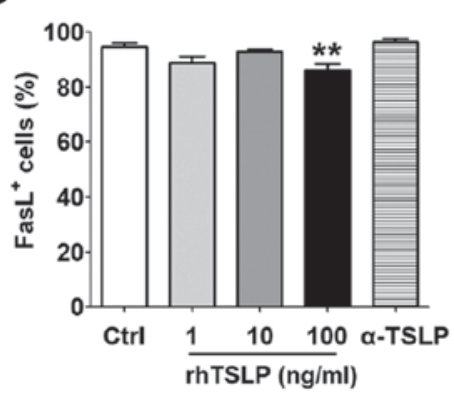

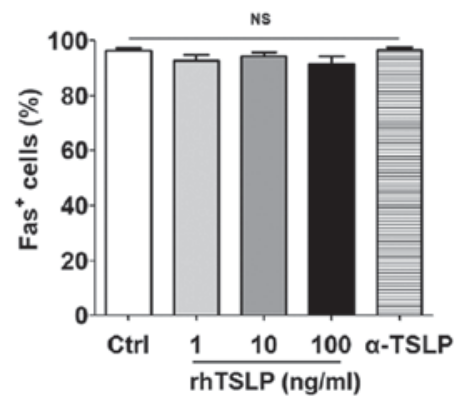

Figure 3. TSLP increases the number of Bcl-2+ ESCs without affecting the expression Fas or FasL in ESCs. (A and B) Expression of Bcl-2 in ESCs was detected by FCM following treatment with rhTSLP $(0,1,10$ or $100 \mathrm{ng} / \mathrm{ml})$ or $\alpha \mathrm{TSLP}(0.25 \mu \mathrm{g} / \mathrm{ml})$. (C) Expression of Fas and FasL in ESCs was detected within ESCs under the same processing conditions. The data are expressed as the mean \pm standard error of the mean. ${ }^{*} \mathrm{P}<0.05$ or ${ }^{* *} \mathrm{P}<0.01$ vs. Ctrl group. Bcl-2, B-cell lymphoma 2; Ctrl, control; ESCs, endometrial stromal cells from patients with endometriosis; FasL, Fas ligand; NS, not significant; rhTSLP, recombinant human thymic stromal lymphopoietin; $\alpha$ TSLP, anti-human TSLP neutralizing antibody.

compared with the control group $(\mathrm{P}<0.05)$ (Fig. 3B). On the contrary, the number of Bcl-2+ ESCs was significantly reduced when $\alpha$ TSLP was applied to neutralize TSLP in the culture system compared with in the control $(\mathrm{P}<0.01)$. The $100 \mathrm{ng} / \mathrm{ml}$ rhTSLP treatment group exhibited a significantly decreased number of $\mathrm{FasL}^{+}$ESCs $(\mathrm{P}<0.01)$; however, no significance 


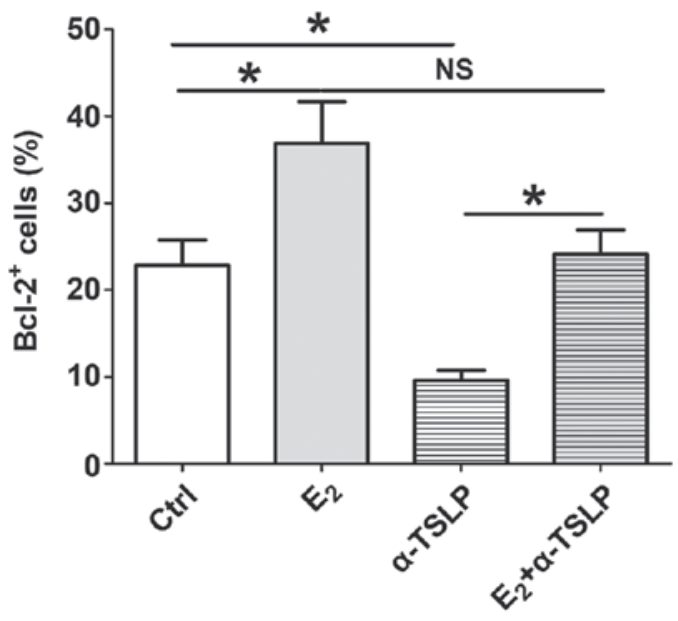

Figure 4. Estrogen increases the number of $\mathrm{Bcl}-2^{+} \mathrm{ESCs}$ by inducing TSLP secretion. Following $\mathrm{E}_{2}$ or $\alpha \mathrm{TSLP}$ treatment or in combination for $48 \mathrm{~h}$, the expression of Bcl-2 in ESCs was detected via flow cytometry. The data are expressed as the mean \pm standard error of the mean. ${ }^{*} \mathrm{P}<0.05$. Ctrl, control; Bcl-2, B-cell lymphoma 2; $\mathrm{E}_{2}, 17-\beta$ estradiol; ESCs, endometrial stromal cells from patients with endometriosis; NS, not significant; TSLP, thymic stromal lymphopoietin; $\alpha$ TSLP, anti-human TSLP neutralizing antibody.

was observed in $\mathrm{Fas}^{+}$ESCs between these groups (Fig. 3C). Additionally, applying $\alpha$ TSLP did not exhibit notable effects on the number of $\mathrm{Fas}^{+}$and FasL ${ }^{+}$ESCs (Fig. 3C). These results indicated that TSLP might inhibit ESC apoptosis probably by promoting Bcl-2 expression in ESCs rather than via the Fas/FasL pathway.

Estrogen increases the number of $\mathrm{Bcl}-2^{+}$ESCs by inducing the secretion of TSLP. Following treatment with $\mathrm{E}_{2}\left(10^{-7} \mathrm{M}\right)$ and/or $\alpha$ TSLP for $48 \mathrm{~h}$, ESCs were obtained for FCM analysis to detect the expression of Bcl-2. As presented in Fig. 4, $\mathrm{E}_{2}$ induced a significant increase in the number of Bcl-2 $2^{+} \mathrm{ESCs}$ compared with in the control $(\mathrm{P}<0.05)$. This effect was notably inhibited when ESCs were incubated with $\alpha$ TSLP within the $\mathrm{E}_{2}$ treatment system; however, no significant difference was reported compared with in the control and $\mathrm{E}_{2}$ treatment groups. As aforementioned, applying $\alpha$ TSLP to the ESC culture system significantly suppressed the number of Bcl-2+ ESC (Fig. 3B); however, in the presence of $E_{2}$, the inhibitory effects of $\alpha$ TSLP were significantly reversed $(\mathrm{P}<0.05)$ (Fig. 4). The results suggested that estrogen could promote TSLP secretion in ESCs, resulting in an increase of Bcl-2 expression and inhibition of ESC apoptosis.

Estrogen-TSLP suppresses the apoptosis of ESCs. To investigate the effect of estrogen and TSLP on the early apoptotic rate of ESCs, cells treated with $\mathrm{E}_{2}$ and/or $\alpha$ TSLP were obtained for FCM analysis (Fig. 5A). Treatment with exogenous $\mathrm{E}_{2}$ led to a significant decrease in the apoptotic rate of ESCs $(\mathrm{P}<0.05)$ and neutralized TSLP resulted in a significantly increased apoptotic rate compared with in the control $(\mathrm{P}<0.001)$ (Fig. 5B). In the $\mathrm{E}_{2}$ and $\alpha \mathrm{TSLP}$ combined treatment group, the apoptotic rate was significantly reduced compared with in the $\alpha$ TSLP group $(\mathrm{P}<0.001)$ (Fig. 5B), but notably increased compared with in the $\mathrm{E}_{2}$ group. This suggested that the respective antiapoptotic and proapoptotic effects of $\mathrm{E}_{2}$ and $\alpha$ TSLP were reduced when combined.

\section{Discussion}

Accumulating studies have reported the importance of eutopic endometrium dysfunction in the pathogenesis of EMS (14,18-21). According to the implantation theory, intrinsic factors of the eutopic endometrium in women with EMS, including aberrantly produced cytokines, growth factors and specific cancer-associated molecules, may be transported to the endometriotic implants and contribute to abnormal cell survival in ectopic tissues $(14,18,19)$. In the eutopic endometrium of women with EMS, the physiological increase in the apoptotic rate in the late secretory phase was not observed, indicating that the imbalance of apoptosis and proliferation may contribute to the implanting of the eutopic endometrium and the survival of the ectopic endometrium (22). In the present study, the apoptosis of eutopic endometrium in the presence of a specific estrogen at high concentrations was investigated to determine the essential role of eutopic endometrium in the occurrence and development of EMS.

Estrogen induces the development of the ectopic endometrium and induce alterations in estrogen signaling (23). The expression levels of ER $\alpha$ and ER $\beta$ of the ectopic endometrial tissues differ to that of eutopic tissues, which possess markedly higher expression levels of $\operatorname{ER} \beta(23,24)$. Via the exogenous addition of estrogen to the ectopic ESC culture system, estrogen was reported to suppress the apoptosis of ESCs, which was associated with promotion of Bcl-2 expression in the present study. As the gene of a typical apoptosis-associated molecule, Bcl-2 may be considered as a novel proto-oncogene that inhibits cell death by regulating mitochondrial membrane function (25). Upregulated Bcl-2 protein expression has been observed in ESCs of ovarian EMS compared with eutopic ESCs from women with and without EMS (26).

TSLP is a cytokine with structural and functional similarities to members of the hematopoietin family (10). The functional and high affinity TSLP receptor (TSLPR) complex is a heterodimer of TSLPR $\alpha$ and IL-7 receptor- $\alpha(27,28)$. TSLP has previously been reported as a contributory factor to a variety of cancers, including pancreatic and breast cancers, which have been associated with Th2-related chronic inflammation $(29,30)$. Consistent with previous studies $(13,14)$, the present study demonstrated that ESCs from patients with EMS possess basal TSLP secretion levels in the absence of estrogen. The limitation for the present study is the lack of analysis of TSLP secretion by ESCs from the control without EMS. Here, it was demonstrated that TSLP secretion was promoted in a dose-dependent manner in response to estrogen treatment. Additionally, TSLP was reported to the affect the mitochondria-dependent intrinsic apoptosis pathway (31). It has been demonstrated that TSLP promotes lymphocyte survival, which was accompanied with increased Bcl-2 expression $(32,33)$. In ESCs, rhTSLP was observed to decrease the apoptotic rate and promote Bcl-2 expression within ESCs in the present study. Inhibition of TSLP via a TSLP neutralizing antibody revealed that the apoptotic rate of ESCs increased and the number of Bcl-2 $2^{+}$ESCs decreased; however, that of Fas/FasL ${ }^{+}$ESCs were notably unaltered in the present study. This suggested that endogenous TSLP may regulate ESC apoptosis in an autocrine manner associated with Bcl-2, but not Fas/FasL (Fig. 6). Therefore, these results indicate that endogenous and 
A

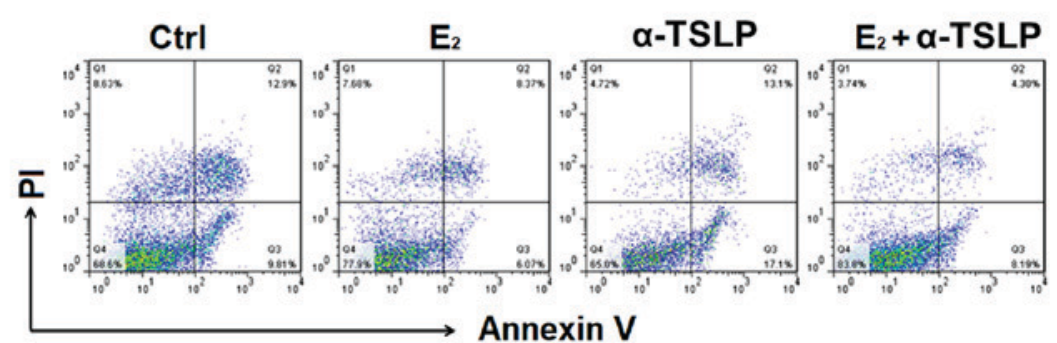

B

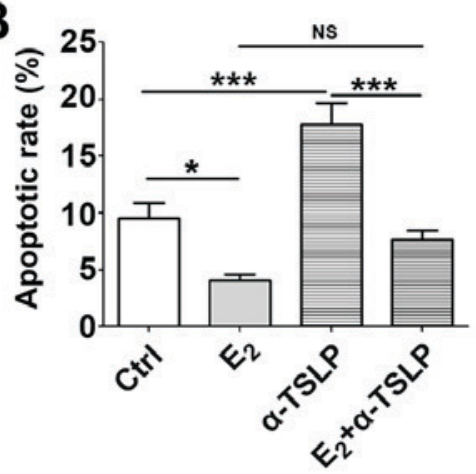

Figure 5. Estrogen and TSLP restricts the apoptosis of ESCs. (A) Following $\mathrm{E}_{2}$ or $\alpha \mathrm{TSLP}$ treatment or in combination for $48 \mathrm{~h}$, the apoptosis of ESCs was determined by flow cytometry. (B) Apoptotic rate of cells. The data are expressed as the mean \pm standard error of the mean. ${ }^{*}<<0.05$ or ${ }^{* * *} \mathrm{P}<0.001$. Ctrl, control; $\mathrm{E}_{2}, 17-\beta$ estradiol; ESCs, endometrial stromal cells from patients with endometriosis; NS, not significant; TSLP, thymic stromal lymphopoietin; $\alpha \mathrm{TSLP}$, anti-human TSLP neutralizing antibody.

exogenous TSLP may inhibit ESC apoptosis by upregulating the expression of Bcl-2.

In the present study, $\mathrm{E}_{2}$ inhibited the apoptosis of ESCs mainly by upregulating the anti-apoptosis protein Bcl-2. When TSLP was neutralized in the presence of estrogen, upregulated Bcl-2 and the reduced apoptotic rate induced by estrogen were partly reversed; however, $\alpha$ TSLP did not fully promote the antiapoptotic and pro-Bcl-2 effects of estrogen. This suggested that TSLP secretion may be one of the mechanisms underlying estrogen-inhibited ESC apoptosis. Other signaling pathways may affect ESC apoptosis; however, further investigation is required. The antiapoptotic effects of estrogen are conducted via numerous pathways involving nuclear and extranuclear ER signaling (9). For instance, the activation of extranuclear kinases by the estrogen-ER complex was reported to lead to a rapid non-genomic signaling cascade, resulting in apoptosis inhibition (9).

In conclusion, the present study revealed a novel estrogen-dependent mechanism underlying the suppression of ESCs apoptosis associated with TSLP secretion and Bcl-2 regulation. In addition, endogenous and estrogen-induced production of endometrial TSLP may promote the initiate and development of EMS via apoptosis inhibition. From the present study, estrogen-TSLP axis may be a new therapeutic target for EMS. This study provides evidence that blocking this pathway may promote ESC apoptosis in treating EMS, but this needs to be investigated further.

\section{Acknowledgements}

Not applicable.

\section{Funding}

The present study was supported by the Major Research Program of National Natural Science Foundation of China (NSFC; grant nos. 91542108, 81471513, 31671200, 3160 0735 and 81601354), the Shanghai Rising-Star Program (grant no. 16QA1400800), the Development Fund of Shanghai Talents (grant no. 201557), the Innovation-oriented Science and Technology Grant from NPFPC Key Laboratory of Reproduction Regulation (grant no. CX2017-2), the Program

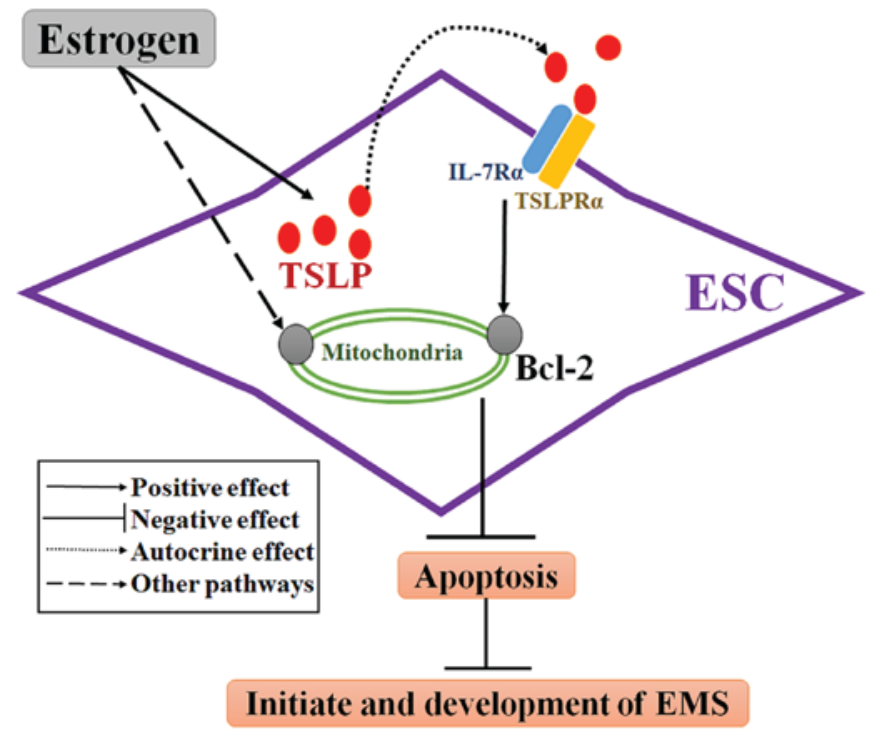

Figure 6. Role of the estrogen/TSLP axis in ESCs. ESCs have basal TSLP secretion and estrogen promotes TSLP secretion of ESCs. Estrogen upregulates Bcl-2 expression to regulate the function of the mitochondrial membrane and then inhibits the apoptosis of ESCs by promoting TSLP expression. Via the antiapoptotic effects of estrogen follow the induction of other signaling pathways. The estrogen/TSLP axis would be a novel mechanism of apoptotic dysfunction within ESCs in EMS, which may be associated with the initiation and development of EMS. Bcl-2, B-cell lymphoma 2; EMS, endometriosis; ESCs, endometrial stromal cells from patients with EMS; IL-7R $\alpha$, interleukin-7 receptor- $\alpha$; TSLP, thymic stromal lymphopoietin; TSLPR $\alpha$, TSLP receptor $\alpha$.

for Zhuoxue of Fudan University, the Natural Science Foundation of Jiangsu Province (grant no. BK20160128), and the Fundamental Research Funds for the Central Universities (grant no. 021414380180).

\section{Availability of data and materials}

The datasets used and/or analyzed during the current study are available from the corresponding author on reasonable request.

\section{Authors' contributions}

H-LY and K-KC conducted all experiments and prepared the figures and the manuscript. JM, W-JZ and L-BL conducted 
flow cytometry analysis. LY, YM, M-YW, S-YH and Z-ZL collected the samples and performed cell isolation. J-FY collected the clinical information and analyzed the data. D-JL assisted in the study design and critically revised the manuscript for important intellectual content. M-QL designed the study, supervised the project and edited the manuscript. All the authors were involved in writing the manuscript.

\section{Ethics approval and consent to participate}

The present study was approved by the Research Ethics Committee in Hospital of Obstetrics and Gynecology, Fudan University. Written informed consent was obtained from all patients prior to enrollment within the study.

\section{Patient consent for publication}

Not applicable.

\section{Competing interests}

The authors declare that they have no competing interests.

\section{References}

1. Kennedy S, Bergqvist A, Chapron C, D'Hooghe T, Dunselman G, Greb R, Hummelshoj L, Prentice A and Saridogan E; ESHRE Special Interest Group for Endometriosis and Endometrium Guideline Development Group: ESHRE guideline for the diagnosis and treatment of endometriosis. Hum Reprod 20 2698-2704, 2005.

2. Sampson JA: Metastatic or embolic endometriosis, due to the menstrual dissemination of endometrial tissue into the venous circulation. Am J Pathol 3: 93-110, 1927.

3. Harada T, Kaponis A, Iwabe T, Taniguchi F, Makrydimas G, Sofikitis N, Paschopoulos M, Paraskevaidis E and Terakawa N Apoptosis in human endometrium and endometriosis. Hum Reprod Update 10: 29-38, 2004

4. Dufournet C, Uzan C, Fauvet R, Cortez A, Siffroi JP and Daraï E: Expression of apoptosis-related proteins in peritoneal, ovarian and colorectal endometriosis. J Reprod Immunol 70: 151-162, 2006.

5. Nasu K, Nishida M, Kawano Y, Tsuno A, Abe W, Yuge A, Takai N and Narahara H: Aberrant expression of apoptosis-related molecules in endometriosis: A possible mechanism underlying the pathogenesis of endometriosis. Reprod Sci 18: 206-218, 2011.

6. Rizner TL: Estrogen metabolism and action in endometriosis. Mol Cell Endocrinol 307: 8-18, 2009.

7. Patel BG, Rudnicki M, Yu J, Shu Y and Taylor RN: Progesterone resistance in endometriosis: Origins, consequences and interventions. Acta Obstet Gynecol Scand 96: 623-632, 2017.

8. Pellegrini C, Gori I, Achtari C, Hornung D, Chardonnens E, Wunder $\mathrm{D}$, Fiche $\mathrm{M}$ and Canny GO: The expression of estrogen receptors as well as GREB1, c-MYC, and cyclin D1, estrogen-regulated genes implicated in proliferation, is increased in peritoneal endometriosis. Fertil Steril 98: 1200-1208, 2012.

9. Reis FM, Petraglia F and Taylor RN: Endometriosis: Hormone regulation and clinical consequences of chemotaxis and apoptosis. Hum Reprod Update 19: 406-418, 2013.

10. Rochman Y and Leonard WJ: Thymic stromal lymphopoietin: A new cytokine in asthma. Curr Opin Pharmacol 8: 249-254, 2008.

11. Liu YJ, Soumelis V, Watanabe N, Ito T, Wang YH, Malefyt Rde W, Omori M, Zhou B and Ziegler SF: TSLP: An epithelial cell cytokine that regulates $\mathrm{T}$ cell differentiation by conditioning dendritic cell maturation. Annu Rev Immunol 25: 193-219, 2007.

12. Zhang Y and Jin LP: Effects of TSLP on obstetrical and gynecological diseases. Am J Reprod Immunol 77, 2017.

13. Urata Y, Osuga Y, Izumi G, Takamura M, Koga K, Nagai M, Harada M, Hirata T, Hirota Y, Yoshino O and Taketani $Y$ : Interleukin-1 $\beta$ stimulates the secretion of thymic stromal lymphopoietin (TSLP) from endometrioma stromal cells: Possible involvement of TSLP in endometriosis. Hum Reprod 27: 3028-3035, 2012.
14. Chang KK, Liu LB, Li H, Mei J, Shao J, Xie F, Li MQ and Li DJ: TSLP induced by estrogen stimulates secretion of MCP-1 and IL-8 and growth of human endometrial stromal cells through JNK and NF-kappaB signal pathways. Int J Clin Exp Pathol 7: 1889-1899, 2014

15. Hayata T, Matsu T, Kawano Y, Matsui N and Miyakawa I: Scanning electron microscopy of endometriotic lesions in the pelvic peritoneum and the histogenesis of endometriosis. Int J Gynaecol Obstet 39: 311-319, 1992.

16. Moen MH and Halvorsen TB: Histologic confirmation of endometriosis in different peritoneal lesions. Acta Obstet Gynecol Scand 71: 337-342, 1992.

17. Chang KK, Liu LB, Jin LP, Zhang B, Mei J, Li H, Wei CY, Zhou WJ, Zhu XY, Shao J, et al: IL-27 triggers IL-10 production in Th17 cells via a c-Maf/ROR $\gamma \mathrm{t} / \mathrm{Blimp}-1$ signal to promote the progression of endometriosis. Cell Death Dis 8: e2666, 2017.

18. Yu JJ, Sun HT, Zhang ZF, Shi RX, Liu LB, Shang WQ, Wei CY, Chang KK, Shao J, Wang MY and Li MQ: IL15 promotes growth and invasion of endometrial stromal cells and inhibits killing activity of NK cells in endometriosis. Reproduction 152: 151-160, 2016.

19. Carvalho L, Podgaec S, Bellodi-Privato M, Falcone T and Abrão MS: Role of eutopic endometrium in pelvic endometriosis. J Minim Invasive Gynecol 18: 419-427, 2011.

20. Mei J, Zhu XY, Jin LP, Duan ZL, Li DJ and Li MQ: Estrogen promotes the survival of human secretory phase endometrial stromal cells via CXCL12/CXCR4 up-regulation-mediated autophagy inhibition. Hum Reprod 30: 1677-1689, 2015.

21. Zhang L, Liu Y, Xu Y, Wu H, Wei Z and Cao Y: The expression of the autophagy gene beclin-1 mRNA and protein in ectopic and eutopic endometrium of patients with endometriosis. Int J Fertil Steril 8: 429-436, 2015.

22. Szymanowski K: Apoptosis pattern in human endometrium in women with pelvic endometriosis. Eur J Obstet Gynecol Reprod Biol 132: 107-110, 2007.

23. Bulun SE, Monsavais D, Pavone ME, Dyson M, Xue Q, Attar E, Tokunaga $\mathrm{H}$ and Su EJ: Role of estrogen receptor- $\beta$ in endometriosis. Semin Reprod Med 30: 39-45, 2012.

24. Bukulmez O, Hardy DB, Carr BR, Word RA and Mendelson CR: Inflammatory status influences aromatase and steroid receptor expression in endometriosis. Endocrinology 149: 1190-1204, 2008.

25. Leibowitz B and Yu J: Mitochondrial signaling in cell death via the Bcl-2 family. Cancer Biol Ther 9: 417-422, 2010.

26. Nishida M, Nasu K, Ueda T, Fukuda J, Takai N and Miyakawa I: Endometriotic cells are resistant to interferon-gamma-induced cell growth inhibition and apoptosis: A possible mechanism involved in the pathogenesis of endometriosis. Mol Hum Reprod 11: 29-34, 2005.

27. Park LS, Martin U, Garka K, Gliniak B, Di Santo JP, Muller W, Largaespada DA, Copeland NG, Jenkins NA, Farr AG, et al: Cloning of the murine thymic stromal lymphopoietin (TSLP) receptor: Formation of a functional heteromeric complex requires interleukin 7 receptor. J Exp Med 192: 659-670, 2000.

28. Zhong $\mathrm{J}$ and Pandey A: Site-directed mutagenesis reveals a unique requirement for tyrosine residues in IL-7R $\alpha$ and TSLPR cytoplasmic domains in TSLP-dependent cell proliferation. BMC Immunol 11: 5, 2010.

29. De Monte L, Reni M, Tassi E, Clavenna D, Papa I, Recalde H, Braga M, Di Carlo V, Doglioni C and Protti MP: Intratumor T helper type 2 cell infiltrate correlates with cancer-associated fibroblast thymic stromal lymphopoietin production and reduced survival in pancreatic cancer. J Exp Med 208: 469-478, 2011.

30. Pedroza-Gonzalez A, Xu K, Wu TC, Aspord C, Tindle S, Marches F, Gallegos M, Burton EC, Savino D, Hori T, et al: Thymic stromal lymphopoietin fosters human breast tumor growth by promoting type 2 inflammation. J Exp Med 208: 479-490, 2011.

31. Ly JD, Grubb DR and Lawen A: The mitochondrial membrane potential (deltapsi(m)) in apoptosis; an update. Apoptosis 8: $115-128,2003$.

32. Pu HH, Duan J, Wang Y, Fan DX, Li DJ and Jin LP: Thymic stromal lymphopoietin promotes the proliferation of human trophoblasts via phosphorylated STAT3-mediated c-Myc upregulation. Placenta 33: 387-391, 2012.

33. Kitajima M, Lee HC, Nakayama T and Ziegler SF: TSLP enhances the function of helper type 2 cells. Eur J Immunol 41: 1862-1871, 2011.

This work is licensed under a Creative Commons Attribution-NonCommercial-NoDerivatives 4.0 International (CC BY-NC-ND 4.0) License. 